\title{
Lichenoid Skin Reaction in a Patient on Anti-Tubercular Drugs: a Rare Case Report
}

Ruchi Arora SACHDEVA ${ }^{1}$, Sachet DAWAR ${ }^{1}$, Sunil NAGAR ${ }^{1}$, Deepali PARASHAR ${ }^{1}$

Abstract

First line anti-tubercular drugs are known to cause various adverse drug reactions including cutaneous reactions. We report a rare case of anti-tubercular treatment (ATT) induced lichenoid skin reaction in a 58 years old female with no prior history of rashes. She was put on fixed dose combination (FDC) tablets of first line anti-tuberculosis drugs and developed reaction one month after initiation of therapy.

Keywords: lichenoid skin reaction, anti-tubercular treatment, fixed dose combination.

\section{INTRODUCTION}

Lichenoid skin reactions are rare cutaneous manifestation to develop in the patients on antitubercular drug $^{1}$. The spectrum of cutaneous adverse drug reaction associated with ATT include various syndromes like Stevens - Johnson syndrome, lichenoid skin reaction, DRESS syndrome, and drug hypersensitivity syndro$\mathrm{me}^{2}$. Fixed dose combination of antitubercular drug regimen was introduced under pilot project of 99 DOTS earlier in 2013 by Government of India. After a huge success in the outcomes, FDC was introduced for all ATT drug regimen under Directly Observed Treatment Short-course (DOTS) in 2015.
Each tablet of FDC consists of Isoniazid (H) $75 \mathrm{mg}$, Rifampicin (R) 150mg, Ethambutol (E) $275 \mathrm{mg}$ and Pyrazinamide $(Z) 400 \mathrm{mg}$. The treatment is based on the weight bands. Daily FDC tablets are provided to the patients by healthcare provider, which has shown good adherence, less misuse of drugs and easy treatment delivery. Common adverse effects of ATT are nausea, vomiting, flushing, hyperuricemia and peripheral neuritis. Rarely, skin rashes and photosensitivity are reported during treatment ${ }^{4,5}$. Thus, this case of lichenoid skin reaction has been found to be rare in the relevant scientific literature.
${ }^{1}$ Department of Respiratory Medicine, ESIC Medical College \& Hospital, Faridabad, Haryana, India

\section{Corresponding author.}

Ruchi Arora SACHDEVA, Department of Respiratory Medicine, ESIC Medical College \& Hospital, NH-3, NIT, Faridabad-121012, Haryana, India.

E-mail:drruchiarorasachdevaesic@gmail.com 


\section{CASE REPORT}

In August 2019, a 58 year old female patient of Faridabad district, belonging to a lower middle class family presented to the Respiratory Medicine Out-Patient Department (OPD) at ESIC Medical College and Hospital, Faridabad, Haryana with chief complaints of cough with expectoration and fever for three weeks. She also reported loss of appetite and weight loss. She did not have any significant past medical history. Chest radiograph was suggestive of right upper lobe collapse (Figure 1). Her sputum samples were examined as per revised national tuberculosis control program (RNTCP) guidelines by Ziehl-Neelson (ZN) staining method and were found to be negative for acid fast bacilli (AFB). Sputum sample sent for cartridge based nucleic amplification test (CBNAAT) tested positive (Mycobacterium tuberculosis: Detected; Rifampicin resistance: Not detected).

On the basis of above findings, patient was diagnosed as a case of pulmonary tuberculosis. Accordingly, she was referred to the DOTS center of ESIC Medical hospital, Faridabad where she was started on Category I fixed dose combination tablets of ATT as per her weight band in the latest RNTCP guidelines. Category I anti tubercular therapy is of six months duration and includes two months of intensive phase (four drugs: HRZE) and four months of continuation phase (three drugs: HRE), both available in fixed dose combinations.

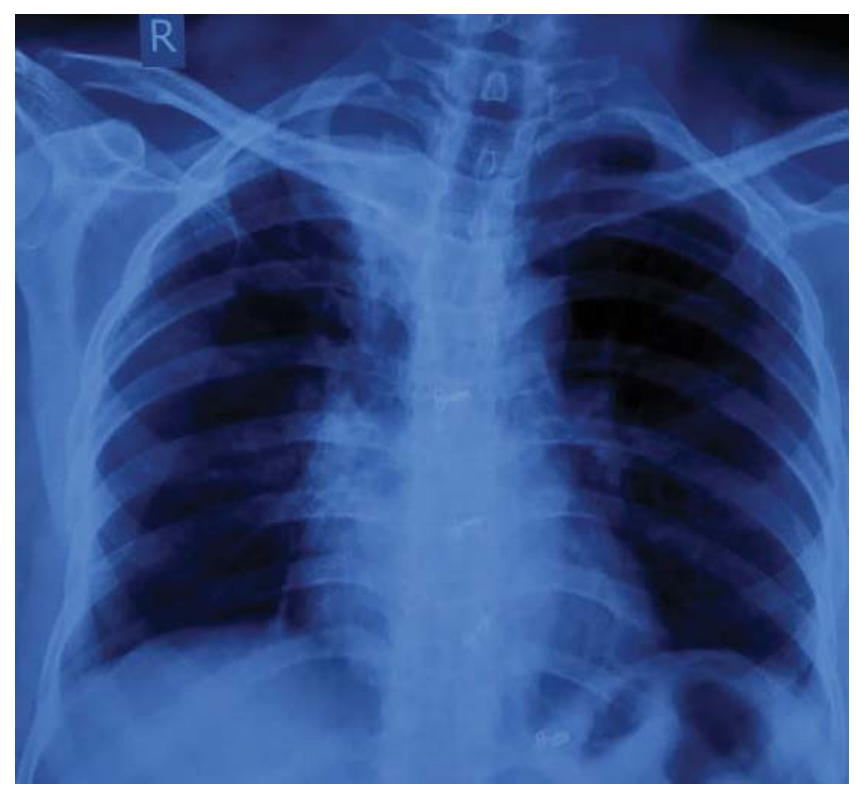

Figure 1. Chest Xray Postero-Anterior View suggestive of right upper lobe collapse.

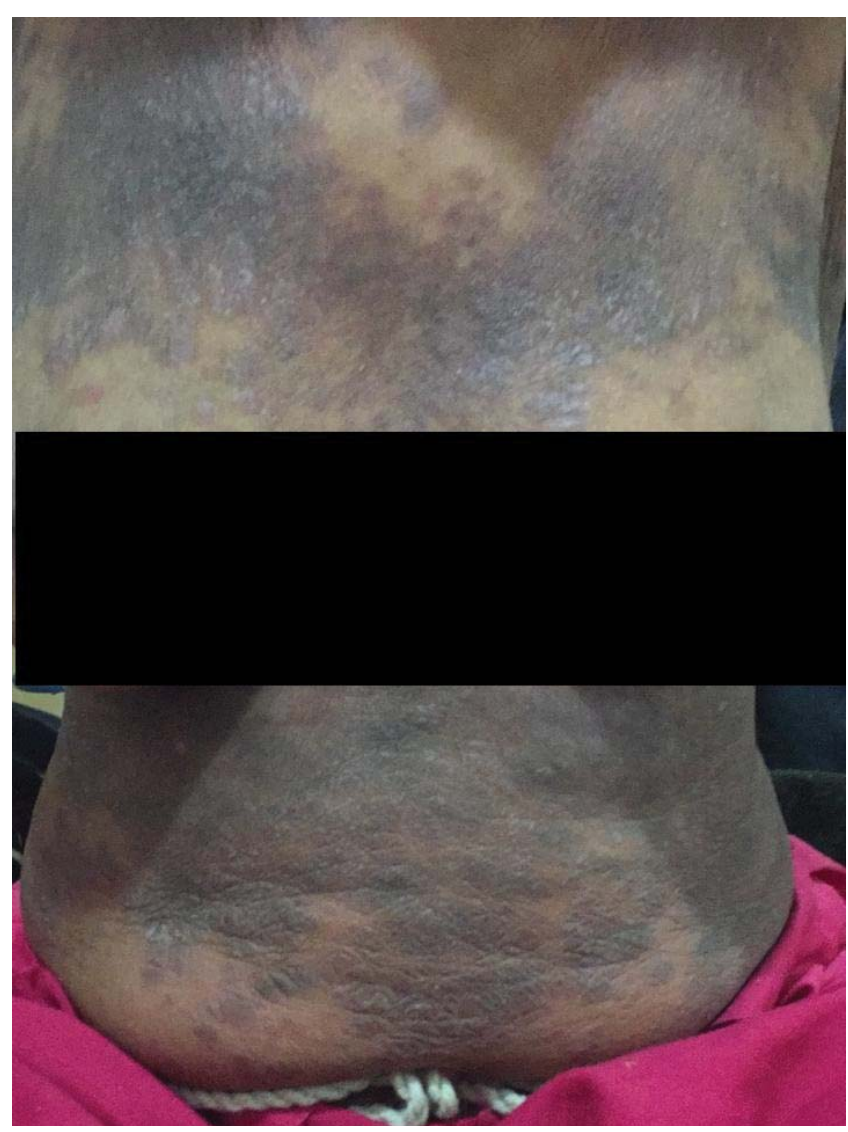

Figure 2. Rash on the trunk.

In the fifth month of ATT patient presented with erythroderma of one month duration. As per history rashes first appeared on her trunk and back (Figure 2, Figure 3) and subsequently on her neck, face, forearm and legs (Figure 4). Rashes were multiple voilaceous papules and plaques, over neck, arm, forearm, trunk and legs as shown in figures. This is the usual pattern observed in Lichenoid drug reactions (LDR) ${ }^{3}$. These rashes were painless but associated with pruritis and hyper-pigmentation. Patient did not give any history of skin lesions in relation to sun exposure in past. Neither history of any other illness or any medications in the past, nor any family history related to the same.

She was thoroughly investigated. Her complete blood profile, blood sugar level, liver \& kidney function tests, serum electrolytes, thyroid profile were within normal limits. HbA1C was $5.4 \%$. Reports were negative for viral serology (HIV, HBsAg, Anti-HCV), VDRL test, anti ds-DNA, anti-histone antibodies, anti-smith antibodies, c-ANCA, p-ANCA, ANA, anti-centromere antibodies and Ro/La antibodies. Skin biopsy showed epidermal hyperkeratosis, presence of 


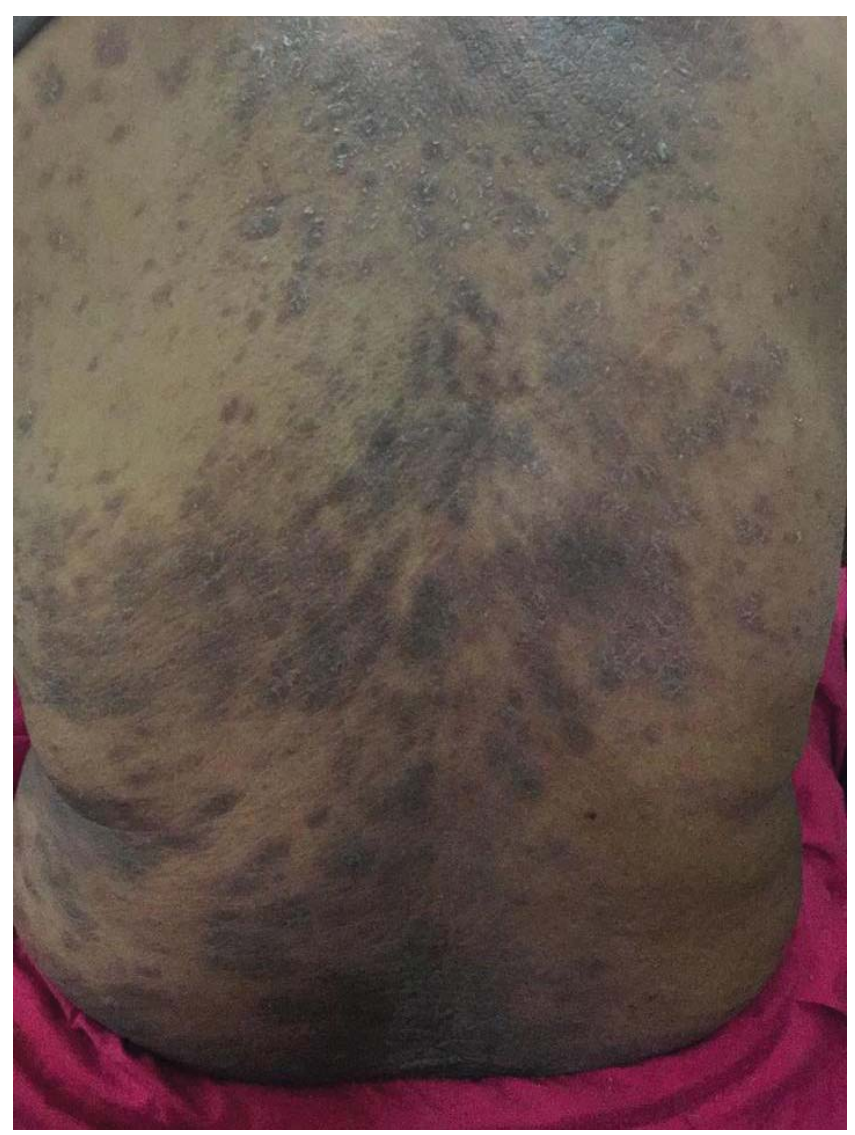

Figure 3. Rash on the back.

peri-epidermal lymphocytic inflammatory infiltrates in the dermis suggestive of lichenoid drug eruptions (Figure 5). A diagnosis of ATT drug induced lichenoid skin reaction was established.

The patient had completed five months of treatment; her follow-up sputum smears were negative for AFB along with clinico-radiological improvement. The ATT drugs were stopped and skin consultation was sought. Patient was put on topical corticosteroids (mometasone cream), calamine lotion and anti-histaminic drugs as per dermatologist's advice. The existing rashes disappeared after supportive treatment and there was no recurrence or new rash anywhere in the body. ATT was re-introduced gradually. She was discharged from the hospital and is on regular follow up on out-patient basis.

\section{DISCUSSION}

Lichenoid drug reaction initially presents as purple itchy papules that later on become confluent and hyperpigmented with continuous exposure to the offending

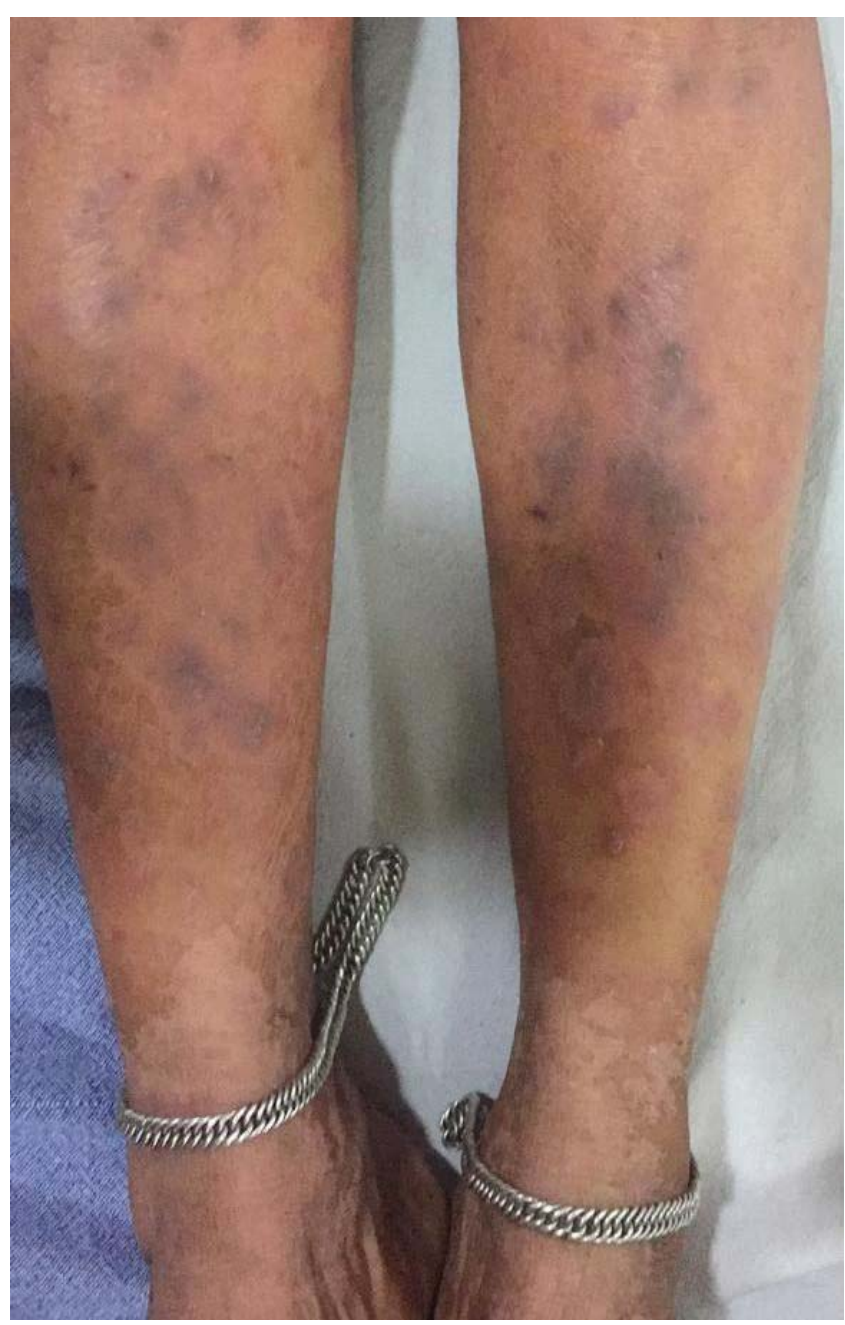

Figure 4. Rash on the lower limbs.

drug. Management of such reactions requires immediate withdrawal of the suspected drug and symptomatic management. ${ }^{9}$ On removal of the offending drug, resolution of rashes occurs but hyper-pigmentation may last for many years. ${ }^{4}$ The first line treatment for LDR is high dose of topical steroids, although no clinical trial supports the belief. Reintroduction of anti-tubercular drugs can be done once the reaction has subsided. Isoniazid and rifampicin are least likely to cause such a reaction and should be reintroduced first. If the initial cutaneous reaction was severe, smaller initial challenge doses should be given ${ }^{10}$.

An autoimmune attack by T-cells on the epidermis is considered the sole cause of drug induced lichenoid eruptions. ${ }^{5}$ Type- $1 \mathrm{~T}$-helper cells (Th-1), cytokines and $\mathrm{CD}^{+} \mathrm{T}$-cells have been shown to play an important role in pathogenesis of non-immediate allergic reacti- 


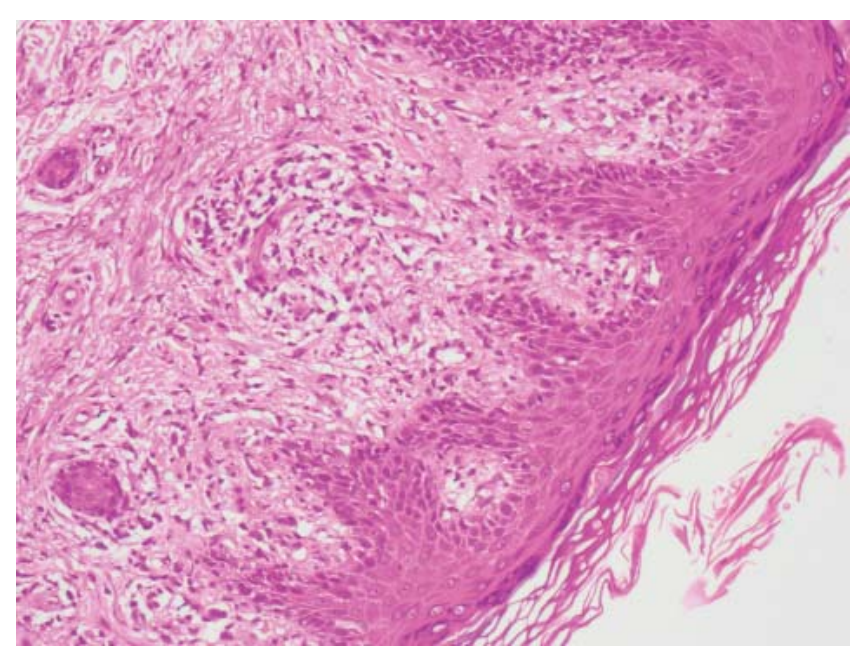

Figure 5. Histopathological appearance showing lymphocytic inflammatory infiltrates and hyper-keratosis.

on due to drugs. Cutaneous reaction to first line ATT drugs are most commonly seen with pyrazinamide (2$5 \%)$, which has been associated with erythema multiforme most frequently6. Rifampicin $(1.2-2 \%)$ is the next commonly associated ATT drug to cause LDR, followed by isoniazid (1-1.2\%) and ethambutol (0.3$1.4 \%)^{7}$. Pyrazinamide is known to cause serious side effects like hepatitis, hyper-uricemia and rash. The incidence of adverse drug reactions (ADR) is higher with $\mathrm{Z}$ as compared to other ATT drugs, and was sex and age related. It is estimated that 1 in 100 patients show some signs of photosensitivity ${ }^{8}$.

In a study done by Horn DL et al, generalized maculo-papular skin rash was a common ADR reported

\section{References}

1. Tan WC, Ong CK, Kang SL, Razak MA. Two years review of cutaneous adverse drug reaction from first line anti-tuberculous drugs. Medical Journal of Malaysia. 2007 Jun 1;62(2):143.

2. KC M. Steven-Johnson Syndrome amongst 9,111 patients undergoing anti-tubercular treatment (a series of 25 cases). Indian J Tuberc. 1978;25(3):141-3.

3. Coulson $\mathrm{IH}$, Benton EC, Ogden S. Diagnosis of skin disease. Rook's Textbook of Dermatology, Ninth Edition. 2016 Jul 15:128.

4. Lindrooth M. Drug Induced Photosensitivity-Drugs and Sun Don't Mix. University of Colorado Hospital, USA. 2010:1-3.

5. Roujeau JC, Stern RS. Severe adverse cutaneous reactions to drugs. New England Journal of Medicine. $1994 \mathrm{Nov}$ 10;331(19):1272-85.

6. Perdu D, Lavaud F, Prevost A, Deschamps F, Cambie MP, Bongrain E, Barhoum K, Kalis B. Erythema multiforme due to pyrazinamide. Allergy. 1996 May;51(5):340-2.

7. Dua R, Sindhwani G, Rawat J. Exfoliative dermatitis to all four first line oral anti-tubercular drugs. Indian J Tuberc. 2010 Jan;57(1):53-6. in investigational trial of ofloxacin (800 $\mathrm{mg}$ a day) and pyrazinamide (1500 $\mathrm{mg}$ a day) prophylaxis against TB. Among 16 ADRs, 3 subjects presented with generalized maculopapular skin rash ${ }^{11}$. There are varieties of other drugs apart from ATT, such as penicillin, allopurinol, minocycline, dapsone, sulfasalazine, phenytoin, phenobarbital, carbamazepine and mexiletine, that have also been associated with drug induced skin eruptions. Also, ATT induced DRESS syndrome (drug reaction with eosinophilia and systemic symptoms) has been reported. DRESS syndrome following ATT is very rare and there are only few case reports in the literature ${ }^{12}$.

\section{CONCLUSION}

Anti-tubercular drugs are known to cause various side effects, of which lichenoid skin reactions are a rare occurrence. As in our case, this ADR caused great cosmetic damage but responded well to withdrawal of ATT and usage of topical steroids. Hence, to conclude any unusual adverse drug reaction has to be recognized early and to be reported in order to reduce mortality and morbidity.

Compliance with ethics requirements: The authors declare no conflict of interest regarding this article. The authors declare that all the procedures and experiments of this study respect the ethical standards in the Helsinki Declaration of 1975, as revised in 2008(5), as well as the national law. Informed consent was obtained from all the patients included in the study.

8. Yee D, Valiquette C, Pelletier M, Parisien I, Rocher I, Menzies D. Incidence of serious side effects from first-line antituberculosis drugs among patients treated for active tuberculosis. American journal of respiratory and critical care medicine. 2003 Jun 1;167(11):1472-7.

9. Bolognia JL, Braverman IM. Skin manifestations of internal disease. In: Braunwald E, Fauci AS, Kasper DL, Hauser SL, Longo $\mathrm{DL}$, Jameson JL, editors. Harrison's Principles of Internal Medicine. 15th ed. McGraw-Hill: New York; 2001. pp. 315-31.

10. Toman K. Toman's Tuberculosis: case detection, treatment, and monitoring: questions and answers. World Health Organization; 2004 Jun 23.

11. Horn DL, Hewlett Jr D, Alfalla C, Peterson S, Opal SM. Limited tolerance of ofloxacin and pyrazinamide prophylaxis against tuberculosis. New England Journal of Medicine. 1994 Apr 28;330(17):1241

12. Kim JH, Jang SH, Kim DH, Park S, Kim DG, Jung KS. A case of DRESS syndrome induced by the antituberculosis drugs, prothionamide, and para-aminosalycilic acid. Annals of Allergy, Asthma \& Immunology. 2013 Feb 1;110(2):118-9. 